\title{
Adenosine Triphosphate
}

National Cancer Institute

\section{Source}

National Cancer Institute. Adenosine Triphosphate. NCI Thesaurus. Code C209.

An adenine nucleotide comprised of three phosphate groups esterified to the sugar

moiety, found in all living cells. Adenosine triphosphate is involved in energy production

for metabolic processes and RNA synthesis. In addition, this substance acts as a

neurotransmitter. In cancer studies, adenosine triphosphate is synthesized to examine its use to decrease weight loss and improve muscle strength. 